\title{
Failed conservative management in a case of placenta percreta lead to total hysterectomy for haemostasis and bladder injury
}

\author{
Victor Gabriel Rugină ${ }^{1}$, Aniela Luminiţa Rugină ${ }^{\star}, 2$, Iulia Ciongradi ${ }^{3}$, Ioan Sârbu ${ }^{3}$, \\ Iolanda Blidaru', Alexandru Cărăuleanu ${ }^{4}$
}

${ }^{1} \mathrm{VV}^{\text {th }}$ Obstetrics Clinic, "Grigore T. Popa" University of Medicine and Pharmacy, lasi, Romania, ${ }^{2} \mathrm{I}^{\text {st }}$ Pediatrics Clinic, "Grigore T. Popa" University of Medicine and Pharmacy, lasi, Romania, ${ }^{3}$ Department of Pediatric and Orthopaedic Surgery, "Grigore T. Popa" University of Medicine and Pharmacy, lasi, ${ }^{4} I^{\text {nd }}$ Obstetrics Clinic, "Grigore T. Popa" University of Medicine and Pharmacy, lasi, Romania

\begin{abstract}
We describe an unfavourable outcome for the conservative management applied to a case of placenta percreta in a 32 year-old woman who referred to our obstetrics department with irregular vaginal bleeding. Her medical history pointed out she had undergone a corporeal caesarean one month before. Ultrasonography revealed a hyperechoic mass in the uterine cavity. After careful investigation by serial values of beta HCG, ultrasound, magnetic resonance imaging (MRI) and exploratory cystoscopy for assessing the degree of placental accretisation we decided to perform an inter-adnexal total hysterectomy with bilateral hypogastric artery ligation. To prevent immediate complications an early diagnosis and a prompt evaluation of the bleeding are important, while a careful follow up is also necessary for predicting any subsequent serious consequences.
\end{abstract}

Keywords: retained products of conception, placenta percreta, abnormal adherent placenta, conservative treatment

\section{Introduction}

Placenta percreta is described as a profound adherence of placental villi to the myometrium. According to the latest epidemiological studies, the number of cases of placenta accreta increased because of higher caesarean rates due to a greater number of accepted indications for caesarean sections, including maternal request [1]. The reported incidence of placenta accreta was 1 in every 533 pregnancies in one report and $2 \%$ in another report [1, 2]. Strong risk factors for

Received: November 2016; Accepted after review: February 2017; Published: March 2017.

*Corresponding author: Aniela Luminiţa Rugină, MD, PhD, $\left.\right|^{\text {st }}$ Pediatrics Clinic, "Grigore T. Popa" University of Medicine and Pharmacy, lasi, 16 Universitatii Street,700115 lasi, Romania.

E-mail: aniela virlan@yahoo.com abnormal placentation include previous caesarean scars and placenta previa. Other known risk factors are multiparity, advanced maternal age, previous dilatation and curettage, a history of manual placenta removal, submucous myoma resulting in atrophy of the endometrium, recurrent abortions, gestational products implanted in the uterine diverticulum, and previous intracavitary radiotherapy. The main danger and most common complication of abnormal placentation is massive postpartum bleeding [2].

Ideally, the diagnosis should be antenatal evaluated in high-risk pregnancies suspected following ultrasound [1]. This could allow for pre-delivery planning to reduce maternal morbidity and mortality. Unfortunately, most cases are identified only at the time of delivery when forcible attempts at manual removal of 
the placenta are unsuccessful [3]. Severe postpartum hemorrhage may result and may lead to complications such as massive transfusion of blood products; disseminated intravascular coagulation (DIC); acute renal failure; infectious morbidities; acute respiratory distress syndrome (ARDS); loss of fertility [1]. Mortality rate may reach 7\% [4].

The conventional treatment for placenta accreta is hysterectomy. However, in cases without massive bleeding and subsequent coagulopathy, a conservative approach may be considered [2]. Conservative approaches include perfusion-decreasing procedures of a radiological or surgical nature. In the former, uterine artery embolization is used, whereas surgical approaches involve bilateral uterine artery ligation and bilateral hypogastric artery ligation procedures [4].

Many situations, such as fertility preservation, medical diseases, lack of access to sufficient blood, and a non-expert surgeon suddenly exposed to placenta accreta in a local hospital, do not support a radical and difficult operation such a total caesarean hysterectomy. We will describe below a case of placenta percreta that initially was successfully managed via conservative uteruspreserving therapy: corporeal caesarean section under iliac balloon inflation for leaving placenta in place after then section of the ombilical cord.

\section{Case report}

A 32 year-old multiparous woman (gravida 6, para 3) presented to our Obstetrics Department, with irregular vaginal bleeding. Her obstetrical history consisted of three caesarean deliveries and three curettages for incomplete abortions. The last caesarean section was performed at Whittington Hospital, London, England at gestational age 32 weeks, for the diagnosis of placenta previa percreta.

Immediately after admission, the patient was informed of the diagnosis and the medical options regarding the advantages and the potential complications of a radical versus conservative treatment, and she accepted the conservative option as a future treatment.
Prior to surgery she had a femoral artery and internal iliac artery catheters placed. A classical corporeal incision was made and the baby was delivered by breech extraction in good condition. The placenta was left in situ and the iliac balloons were inflated. The uterus was closed in three layers. The balloons were deflated after approximately 22 minutes. The estimated loss of blood was $600 \mathrm{ml}$. One pack of red blood cells was transfused intraoperatively at starting Haemoglobin $(\mathrm{Hb})$ of 8.2 $\mathrm{mg} / \mathrm{dl}$, postoperative $\mathrm{Hb}$ was $7.7 \mathrm{mg} / \mathrm{dl}$ and therefore two further units of red blood cells were transfused. Postoperative antibiotic therapy was instituted with Augmentin and Gentamicin. She was discharged at day fifteen post-op, following multiple scans assessing the placenta at and beta HCG value of 2872 IU/L. Two weeks later, she was readmitted with a secondary postpartum hemorrhage. Her $\mathrm{Hb}$ value remained stable at $9.3 \mathrm{mg} / \mathrm{dl}$ and her beta HCG level dropped to $451 \mathrm{IU} / \mathrm{L}$. Ultrasound revealed a lower uterine placental mass $(109 / 74 / 101 \mathrm{~mm})$ with mixed echoes and moderate vascularity.

On postpartum day 46 , she was referred to our obstetrics department with irregular vaginal bleeding. Upon arrival, her serum beta HCG was $68 \mathrm{IU} / \mathrm{L}$, her $\mathrm{Hb}$ was $7.4 \mathrm{mg} / \mathrm{dl}$, Cephalexin prophylaxis was established. Next day she described ongoing mild-to-moderate vaginal bleeding and was found to have an isolated temperature of $38.5^{\circ} \mathrm{C}$. Blood, urinary and vaginal cultures were taken, two units of packed red blood cells were transfused, and oral Cephalexin administration was continued. An abdominal ultrasound (Figure 1) revealed persistent retained placental tissue. The patient consented to continue the conservative treatment. She provided an informed consent with respect to possible complications, including laparotomy and hysterectomy.

The following day, she received a single dose of $75 \mathrm{mg}$ Methotrexate intramuscularly and was set on a course of prophylactic Cephalexin. An abdominal-pelvic MRI and an exploratory cystoscopy were performed in order to confirm the diagnosis of retention of placenta percreta and to establish the degree of the uterine wall and the eventual penetration into the urinary bladder (Figures 2 and 3). 


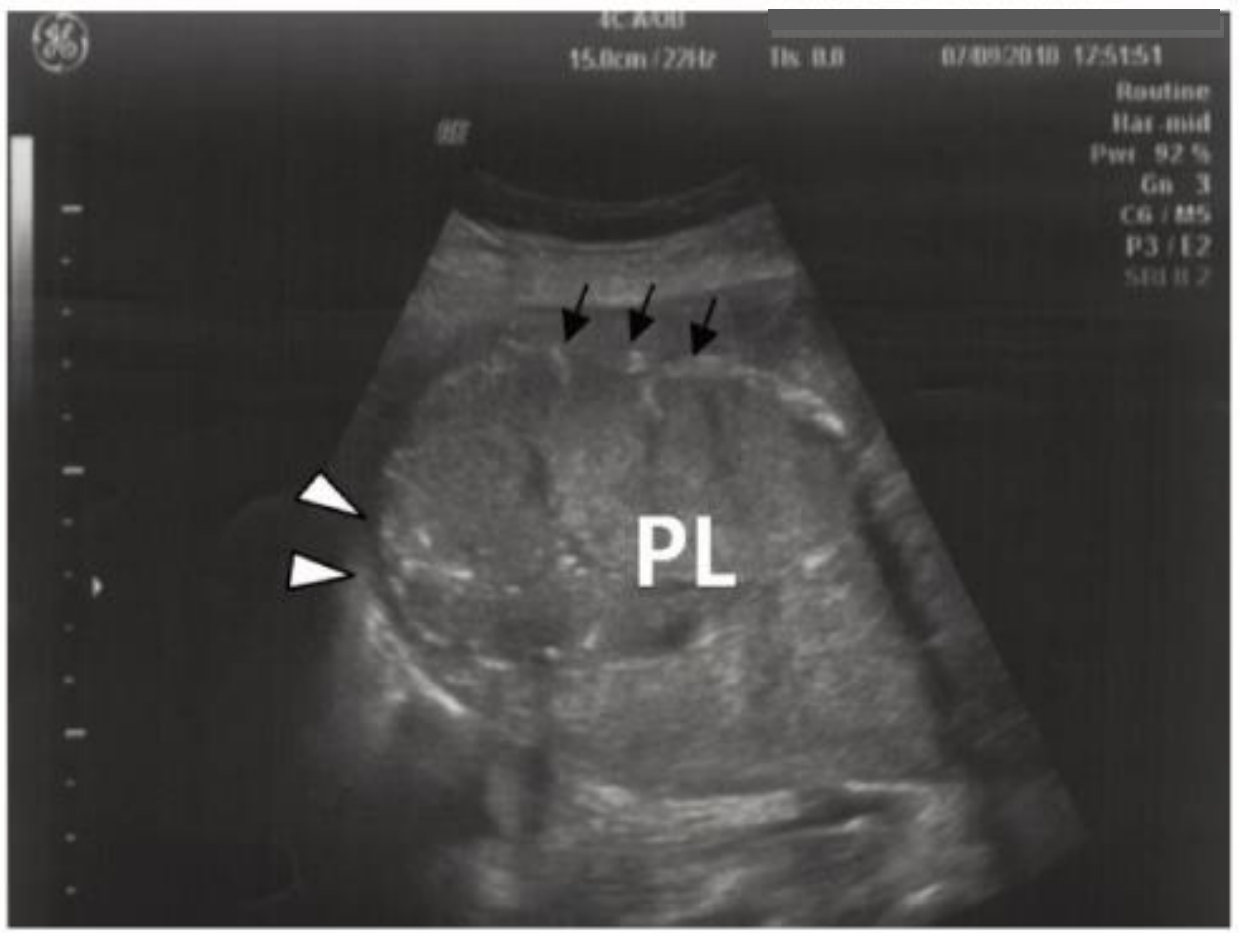

Fig. 1. Transabdominal ultrasound image taken on postpartum day 47: this view of the uterine fundus demonstrates significant persistence of retained placental tissue, progressive disappearance (black arrows)/thinning of the hypoechoic retroplacental zone (white arrows)
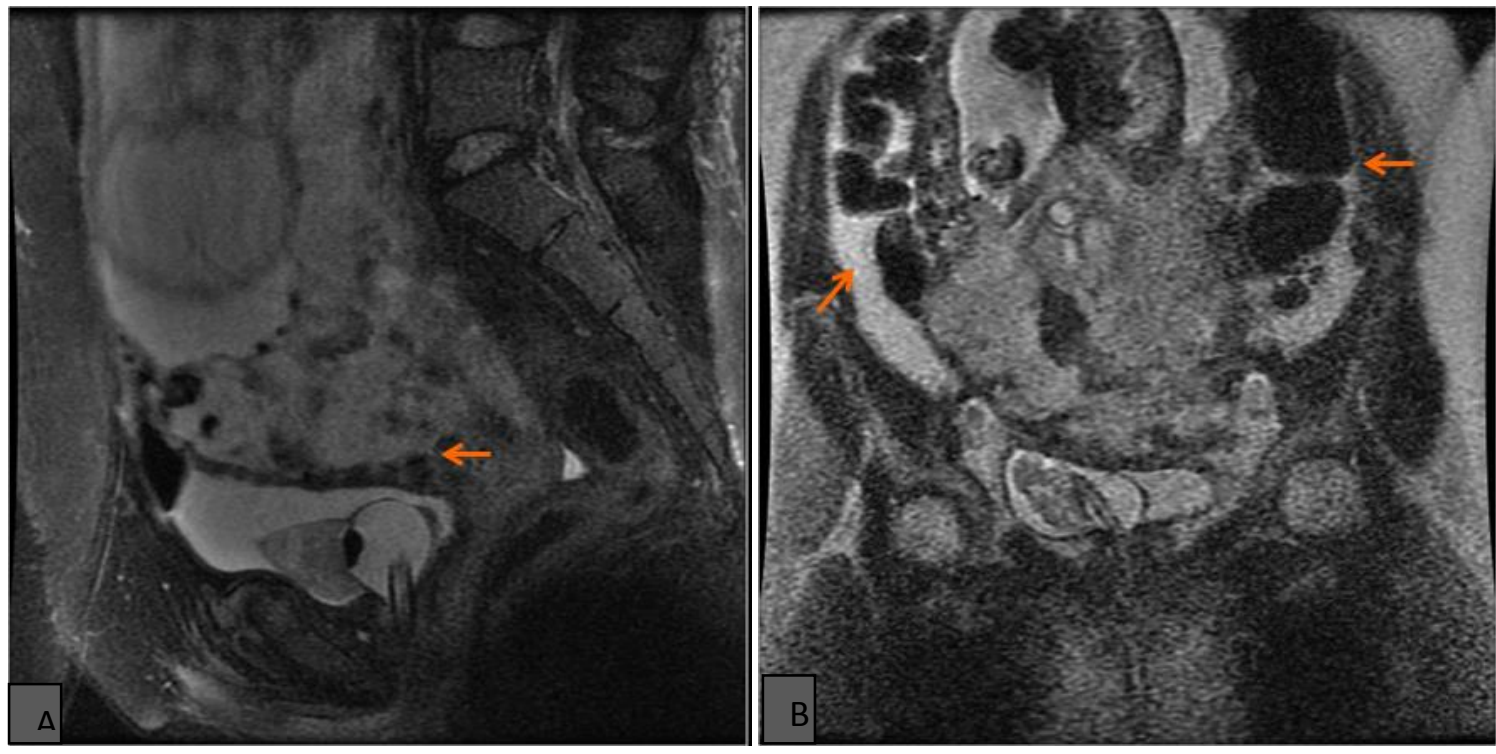

Fig. 2. MRI scan of the patient's abdomen on postpartum day 47 showing the uterus with the retained placenta was demonstrated. A. Hypervascularization in the uterine-urinary bladder wall B. Uterine vessels hypertrophy 


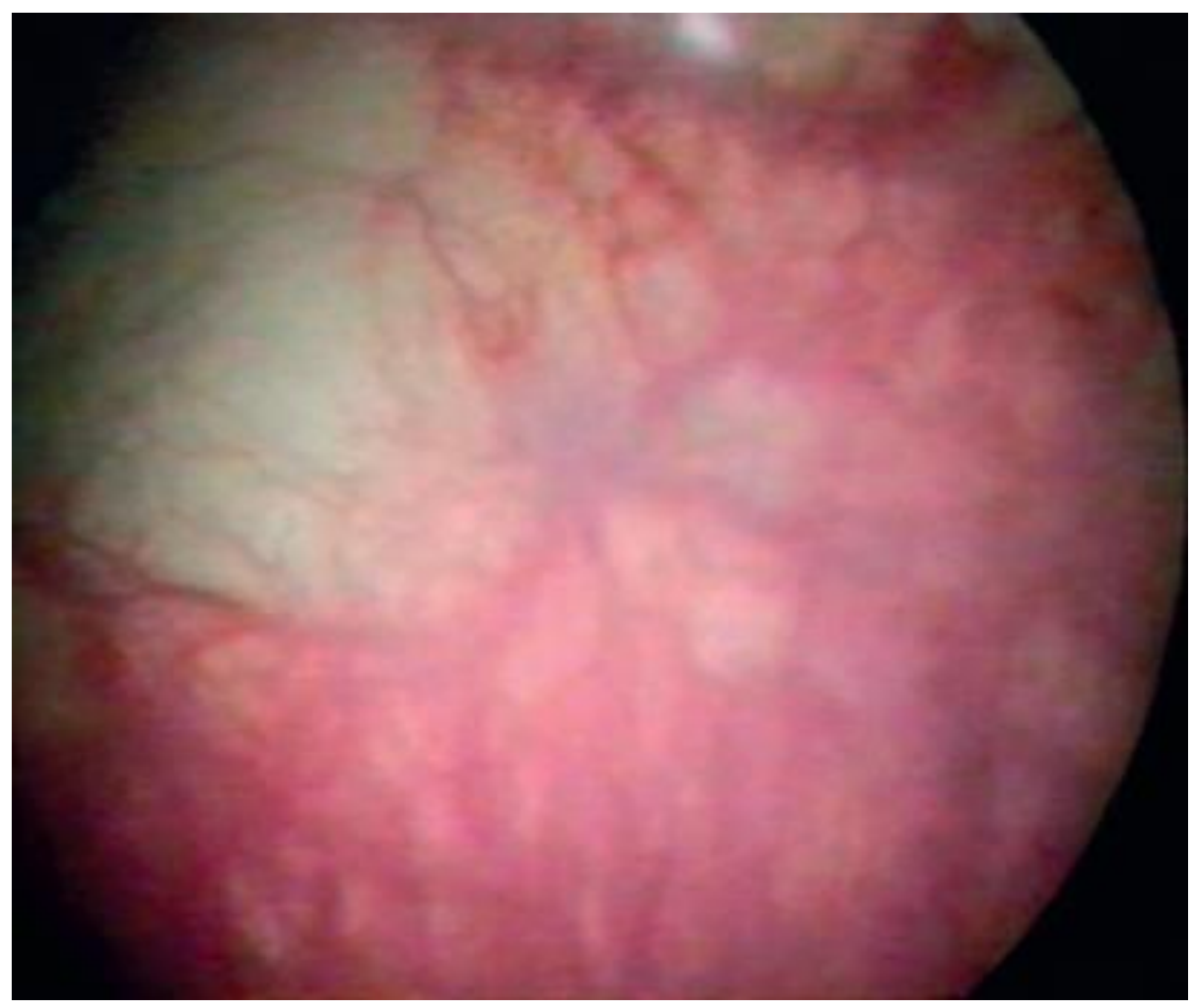

Fig. 3. Exploratory cystoscopy revealing a submucosal bladder vascular network. The integrity of the bladder mucosa is evident, but the extent of vascularization is not seen in detail and information about the uterine serosa-bladder interface is not discernible

On postpartum day 51 , the patient repeated the vaginal bleeding that worsens her general condition and we concluded that the radical treatment was necessary. A complex surgery team consisting of an urologist, one general surgeon and two obstetricians was formed and they performed a total inter-adnexal hysterectomy with the bilateral ligation of the hypogastric arteries. During hysterectomy, the urinary bladder was affected and needed to be rebuilt. One intraperitoneal Douglas drain was left in. The intra-operative blood loss was approximately $500 \mathrm{ml}$, the patient received 2 units of integral blood.

Postoperatively the patient recovered. She received further urinary bladder rest for 7 days. A mild wound infection developed on postoperative day 4 , and antibiotic therapy was supplemented with Gentamicin for E. coli (wound), and non-haemolytic streptococcus (wound).

The patient was finally discharged from hospital in good condition on postoperative day 10. Pathology confirmed the presence of necrotic and infarcted villous tissue in the uterine wall consistent with placenta percreta. Incidentally, the cervix was found to have highgrade dysplasia.

\section{Discussion}

After medical and surgical pregnancy termination, and vaginal or caesarean delivery, there may be partial or complete retention of parts of the placenta or other residual tissues within the uterus. This condition is defined as RPOC, meanwhile, leaving placenta in place as a conservative treatment of placenta accreta is a voluntary therapeutic approach. Both conditions are causes of both primary and secondary postpartum hemorrhage [1]. One of the most important risk factors for RPOC is placenta accreta.

The majority of women with placenta accreta have no symptoms during pregnancy [2].

Patients with conservative treatment for placenta percreta by leaving the placenta in 
place usually presented abdominal pain, bleeding, fever: these clinical symptoms are not specific, but the early diagnosis is critical for directing clinical management of the bleeding and for preventing associated consequences [3].

However prenatal diagnosis of morbidly adherent placenta and its variants can help to reduce maternal/fetal morbidity and mortality. Cheung et al. identified the following ultrasonographic criteria for morbidly adherent placenta: loss/irregularity of the echolucent area between the uterus and the placenta, termed 'clear space'; thinning or interruption of the hyperechoic interface between the uterine serosa and the bladder wall, termed 'bladder line'; presence of turbulent placental lacunae with high-velocity flow $(>15 \mathrm{~cm} / \mathrm{s})$.

Transabdominal 3D power Doppler was used to map the vascularization of the intraplacental and uterine serosa-bladder interface. In particular, the sagittal sections were used to assess the depth of placental tissue neovascularisation and whether this was limited to the basal layer or it affected the entire remaining placental parenchyma [5].

Our experience shows that the first step in all bleeding cases was to evaluate the uterine cavity by ultrasound, but sometimes it was difficult to distinguish between placental tissue and blood clots, however ultrasonography combining Gray Scale-Color Doppler and sonohysterography is the best predictor in women with a suspicion of RPOC. This approach represents a useful diagnostic tool for prompt evaluation and timely treatment as well as for fertility preservation for these women. So a careful investigation, efficient planning management and an accurate follow up are required in patients with retained products of conception to lessen morbidity and to preserve their obstetric outcome [6].

During pregnancy, the uterine arteries contain $90 \%$ of the uterine blood supply. According to a literature review of existing surgical management, in the absence of severe blood loss and subsequent coagulopathy several conservative procedures are suggested. Among those conservative nonsurgical procedures, the occlusion by balloon of aorta and common iliac arteries by puncturing and entering a catheter through the femoral arteries by a radiologist, helps diminish the loss of blood during the caesarean section in case of placenta accreta $[7,8]$.

Theoretically, the proximal occlusion of the large vessels such as the aorta and the common iliac arteries is more effective, but it runs the potential risk of ischemia to the extremities if prolonged occlusion is required $[9,10]$. The use of catheter-based balloon occlusion to thwart massive obstetric hemorrhage was first described by Paull et al [11] in 1995 with the use of an $18 \mathrm{~mm}$ infrarenal aortic balloon. Soon after, Dubois et al [12] described the successful prophylaxis of two patients with placenta percreta using 8.5 $\mathrm{mm}$ occluding balloon catheters placed in the anterior divisions of both internal iliac arteries. Levine et al [13] reported their preliminary findings that transcatheter arterial balloon occlusion procedures did not improve surgical outcomes compared with patients treated without them. However, Kidney et al [14] reported that balloon occlusion of the hypogastric arteries was an effective adjunct to caesarean hysterectomy in an attempt to minimize operative blood loss in patients with abnormal placentation. They suggested that although occlusion of the hypogastric arteries did not halt blood flow to the uterus because there is a rich supply of collaterals, the technique did reduce the pulse pressure distal to the site of occlusion, thus minimizing blood loss during hysterectomy.

Shrivastava et al [15] reported a large case-controlled study of 69 patients who had caesarean hysterectomy performed for placenta accreta. Nineteen patients with preoperative internal iliac artery balloon catheter placement plus hysterectomy were compared with 50 patients who had hysterectomy alone. No significant differences were noted in estimated blood loss. Importantly, three of the $19 \mathrm{BC}$ patients $(15.8 \%)$ had severe complications from catheter placement; one patient was noted to have an internal iliac artery thrombosis and groin hematoma. A second patient had an internal iliac artery dissection with $80-90 \%$ occlusion. A third patient had a femoral artery thrombosis. 
A conservative approach is recommended, even in women who do not want to preserve their fertility, considering the morbidity associated with a caesarean hysterectomy. A Chinese suggested that the average transfusion requirement in caesarean hysterectomy operations was of 10 units of packed red cells and four units of fresh frozen plasma [16]. The emergent and sometimes massive transfusion needs of these women necessitate a large blood bank. Many hospitals cannot support blood component requirements of an emergent caesarean hysterectomy for abnormal placentation [17]. A total caesarean hysterectomy is difficult and it is not chosen in many cases. If extirpative surgery (hysterectomy) is performed for placenta accreta in the lower segment, the technical difficulty associated to total instead of subtotal hysterectomy might result in urologic and hemostasis complications. A total hysterectomy is associated with an increased risk of significant hemorrhage and damage to the urinary and gastrointestinal tract [18].

According to the literature, the overall rate of hysterectomy in patients with placenta accreta who are treated conservatively is $19.3 \%$. Uterine artery ligation or the embolization procedure alone might fail [16].

Postoperative complications reported with the established conservative approach include severe postpartum hemorrhage, postoperative disseminated intravascular coagulopathy, and infection resistant to antimicrobial therapy that may require laparotomy and hysterectomy [19]. Thus far, the results of using preoperative prophylactic internal iliac artery catheterization as an adjuvant treatment to hysterectomy or in cases of conservative management are equivocal, and they are largely limited by the small sample size of the studies. So, there is as yet no effective conservative treatment of placenta accreta, and hysterectomy is the preferred solution for these patients. Therefore, a new conservative treatment approach is required for women who have a strong desire for preserving their future fertility.

Planned caesarean hysterectomy at the time of caesarean is the standard recommended treatment in the United States, but over the past years, interest in conservative management has resurged [6]. Conservative management should be the preferred approach for reducing the morbidity of peripartum hysterectomy and especially for preserving fertility in young women. However subsequent pregnancies after conservative treatment for placenta accreta were mostly successful, a history of placenta accreta is an independent risk factor for recurrent placenta accreta and postpartum hemorrhage [20]. This should be taken into account in order to ensure future safety of pregnancies and deliveries to patients. In fact, with these patients, the site and time of delivery, as well as the surgical approach, can be planned ahead and blood loss thus minimized.

\section{Conclusion}

According to the American College of Obstetricians and Gynaecologists, a caesarean hysterectomy is the treatment of choice for placenta percreta. The presented case report describes one case of initial successful conservative management of placenta percreta (C-section preceded by proximal occlusion of the common iliac artery for decreasing intraoperative bleeding) that needed a radical treatment in the end. However, the conservative treatment's initial success allowed the patient to exit the early postpartum period when she is most susceptible to secondary coagulopathy due to acute uterine bleeding. Also, blood bank shortages in local hospitals and Rh-negative blood group leading to serious danger of hypovolemia might guide surgeons to procedures with less blood loss such as the conservative procedures.

\section{Conflict of interest}

Authors declare no conflicts of interest.

\section{Consent}

Written informed consent was obtained from the patient for publication of this case report and accompanying images. 


\section{References}

1. Grace Tan SE, Jobling TW, Wallace EM, et al. Surgical management of placenta accreta: a 10-year experience. Acta Obstet Gynecol Scand 2013; 92(4):445-450.

2. Kamara M, Henderson JJ, Doherty DA, et al. The risk of placenta accreta following primary elective caesarean delivery: a case-control study. BJOG 2013; 120(7):879-886.

3. Bencaiova G, Burkhardt $T$, Beinder $E$. Abnormal placental invasion experience at 1 center. J Reprod Med 2007; 52(8):709-714.

4. Sentilhes L, Ambroselli C, Kayem G, et al. Maternal outcome after conservative treatment of placenta accreta. Obstet Gynecol 2010; 115(3):526534.

5. Cheung CS, Chan BC. The sonographic appearance and obstetric management of placenta accreta. Int J Womens Health 2012; 4:587-594.

6. American College of Obstetricians and Gynecologists. ACOG Practice Bulletin: Clinical Management Guidelines for ObstetricianGynecologists Number 76, October 2006: postpartum hemorrhage. Obstet Gynecol 2006; 108(4): 1039-1047.

7. Chou MM, Kung HF, Hwang JI, Chen WC, Tseng JJ. Temporary prophylactic intravascular balloon occlusion of the common iliac arteries before caesarean hysterectomy for controlling operative blood loss in abnormal placentation. Taiwan J Obstet Gynecol 2015; 54(5):493.

8. Shahin AY, Farghaly TA, Mohamed SA, et al. Bilateral uterine artery ligation plus B-Lynch procedure for atonic postpartum hemorrhage with placenta accreta. Int J Gynaecol Obstet 2010; 108(3):187-190.

9. Soncini E, Pelicelli A, Larini $P$, et al. Uterine artery embolization in the treatment and prevention of postpartum hemorrhage. Int $J$ Gynaecol Obstet 2007; 96(3):181-185.

10. Knuttinen MG, Jani $A$, Gaba RC, et al. Balloon occlusion of the hypogastric arteries in the management of placenta accreta: a case report and review of the literature. Semin Intervent Radiol 2012; 29:161e8.

11. Paull JD, Smith J, Williams L, et al. Balloon occlusion of the abdominal aorta during caesarean hysterectomy for placenta percreta. Anaesth Intensive Care 1995; 23:731e4.

12. Dubois J, Garel L, Grignon A, et al. Placenta percreta: Balloon occlusion and embolization of the internal iliac arteries to reduce intraoperative blood losses. Am J Obstet Gynecol 1997; 176:723e6.

13. Levine AB, Kuhlman K, Bonn J. Placenta accreta: Comparison of cases managed with and without pelvic artery balloon catheters. J Matern Fetal Neonatal Med 1999;8: 173e6.

14. Kidney DD, Nguyen AM, Ahdoot D, et al. Prophylactic perioperative hypogastric artery balloon occlusion in abnormal placentation. $A m \mathrm{~J}$ Roentgenol 2001; 176:1521e4.

15. Shrivastava V, Nageotte M, Major $C$, et al. Case-control comparison of caesarean hysterectomy with and without prophylactic placement of intravascular balloon catheters for placenta accreta. Am J Obstet Gynecol 2007; 197(4):402e1-5.

16. Lyu B, Chen M, Liu XX. Risk factors of peripartum hysterectomy in placenta previa: a retrospective study of 3840 cases. Zhonghua Fu Chan Ke Za Zhi 2016; 51(7):498-502.

17. Friedman AM, Wright JD, Ananth CV, et al. Population-based risk for peripartum hysterectomy during low- and moderate-risk delivery hospitalizations. Am J Obstet Gynecol 2016; 215(5):640e1-e8.

18. Daney de Marcillac $F$, Lecointre $L$, Guillaume A, et al. Maternal morbidity and mortality associated with conservative management for placenta morbidly adherent (accreta) diagnosed during pregnancy. Report of 15 cases. J Gynecol Obstet Biol Reprod 2016; 45(8):849-858.

19. Wang QM, Liu HL, Dang Q. Acute trophoblastic pulmonary embolism during conservative treatment of placenta accreta: case report and review of literature. Eur $J$ Med Res. 2015; 20:91.

20. Sentilhes L, Kayem G, Ambroselli C, et al. Fertility and pregnancy outcomes following conservative treatment for placenta accreta. Hum Reprod 2010; 25(11):2803-2810. 